\title{
Distributed Weighted-Multidimensional Scaling for Node Localization in Sensor Networks
}

\author{
JOSE A. COSTA, NEAL PATWARI, and ALFRED O. HERO III \\ University of Michigan, Ann Arbor
}

\begin{abstract}
Accurate, distributed localization algorithms are needed for a wide variety of wireless sensor network applications. This article introduces a scalable, distributed weighted-multidimensional scaling (dwMDS) algorithm that adaptively emphasizes the most accurate range measurements and naturally accounts for communication constraints within the sensor network. Each node adaptively chooses a neighborhood of sensors, updates its position estimate by minimizing a local cost function and then passes this update to neighboring sensors. Derived bounds on communication requirements provide insight on the energy efficiency of the proposed distributed method versus a centralized approach. For received signal-strength (RSS) based range measurements, we demonstrate via simulation that location estimates are nearly unbiased with variance close to the Cramér-Rao lower bound. Further, RSS and time-of-arrival (TOA) channel measurements are used to demonstrate performance as good as the centralized maximum-likelihood estimator (MLE) in a real-world sensor network.
\end{abstract}

Categories and Subject Descriptors: C.2.4 [Computer-Communication Networks]: Distributed Systems-Distributed applications; I.5.4 [Pattern Recognition]: Applications-Signal processing General Terms: Algorithms, Performance

Additional Key Words and Phrases: Distributed optimization, multidimensional scaling, node localization, position estimation, sensor networks

\section{INTRODUCTION}

For monitoring and control applications using wireless sensor networks, automatic localization of every sensor in the network will be a key enabling technology. Sensor data must be registered to its physical location to permit deployment of energy-efficient routing schemes, source localization algorithms, and distributed compression techniques. Moreover, for applications such as inventory

This work was partially supported by the National Science Foundation under ITR grant CCR0325571.

Authors' Address: Department of Electrical Engineering and Computer Science, University of Michigan, 1301 Beal Avenue, Ann Arbor, MI 48109-2122; email: jcosta@umich.edu;\{npatwari, hero\}@eecs.umich.edu.

Permission to make digital or hard copies of part or all of this work for personal or classroom use is granted without fee provided that copies are not made or distributed for profit or direct commercial advantage and that copies show this notice on the first page or initial screen of a display along with the full citation. Copyrights for components of this work owned by others than ACM must be honored. Abstracting with credit is permitted. To copy otherwise, to republish, to post on servers, to redistribute to lists, or to use any component of this work in other works requires prior specific permission and/or a fee. Permissions may be requested from Publications Dept., ACM, Inc., 1515 Broadway, New York, NY 10036 USA, fax: +1 (212) 869-0481, or permissions@acm.org.

(C) 2006 ACM 1550-4859/06/0200-0039 $\$ 5.00$ 
management and manufacturing logistics, localization and tracking of sensors are the primary purposes of the wireless network. For large-scale networks of inexpensive, energy-efficient devices, it is not feasible to include GPS capability on every device or to require a system administrator to manually enter all device coordinates. In this article, we consider the location estimation problem for which only a small fraction of sensors have a priori coordinate knowledge, and range measurements between many pairs of neighboring sensors permit the estimation of all sensor coordinates. While angle measurements have also been used for sensor localization, in this article, we limit the discussion to localization based on range measurements.

Two major difficulties hinder accurate sensor location estimation: first, accurate range measurements are expensive; and second, centralized estimation becomes impossible as the scale of the network increases. This article proposes a distributed localization algorithm, based on a weighted version of multidimensional scaling (MDS), which naturally incorporates local communication constraints within the sensor network. Its key features are:

(1) A weighted cost function, which allows range measurements that are believed to be more accurate to be weighted more heavily.

(2) An adaptive neighbor selection method, which avoids the biasing effects of selecting neighbors based on noisy range measurements.

(3) A majorization method, which has the property that each iteration is guaranteed to improve the value of the cost function.

Simulation results and experimental channel measurements show that even when using only a small number of range measurements between neighbors and relying on fading-prone received signal-strength (RSS), the proposed algorithm can be nearly unbiased with performance close to the Cramér-Rao lower bound.

\subsection{Sensor Localization Requirements}

For a network of thousands or even millions of sensors, the large scale precludes centralized location estimation. Sending pair-wise range measurements from each sensor to a single point and then sending back estimated device coordinates would overwhelm the capacity of low-bandwidth sensor networks and waste energy. Decentralized algorithms are vital for limiting communication costs (which are usually much higher than computation costs), as well as for balancing the communication and computational load evenly across the sensors in the network. Furthermore, when a sensor moves, the ability to recalculate location locally rather than globally will result in energy savings which, over time, may dramatically extend the lifetime of the sensor network.

Sensor energy is also conserved by limiting transmission power. For a given channel between a pair of wireless sensors, the signal to noise ratio (SNR) of the received signal can be improved by increasing the transmit power. Range measurement accuracy improves at higher SNR [Caffery Jr. and Stuber 1998; Kim et al. 2002], thus imposing a tradeoff between energy cost and accuracy. There is also a tradeoff between device cost and range accuracy: using 
ultrawideband (UWB) [Fleming and Kushner 1995; Correal et al. 2003] or hybrid ultrasound/RF techniques [Girod et al. 2002] can achieve accuracies on the order of centimeters, but at the expense of high device and energy costs. Alternatively, very inexpensive wireless devices can measure RF RSS just by listening to network packet traffic, but range estimates from RSS incur significant errors due to channel fading. All range measurements tend to degrade in accuracy with increasing distance. In particular, RSS-based range measurements experience errors whose variance is proportional to the actual range. Accurate localization algorithms must take into account the range dependence of the ranging variance.

Finally, measurement of ranges between every pair of devices would require $\mathrm{O}\left(n^{2}\right)$ measurements for $n$ sensors. The distributed weighted-multidimensional scaling (dwMDS) algorithm reduces measurement costs by requiring range measurements only between a small number of neighboring sensors.

\subsection{Multidimensional Scaling}

The goal of multidimensional scaling is to find a low dimensional representation of a group of objects (e.g., sensor positions), such that the distances between objects fit as well as possible a given set of measured pairwise "dissimilarities" that indicate how dissimilar objects are (e.g., inter-sensor RSS). MDS has found many applications in chemical modeling, economics, sociology, political science and, especially, mathematical psychology and behavioral sciences [Cox and Cox 1994]. More recently, MDS has been used by the machine learning community for manifold learning [Tenenbaum et al. 2000]. In the sensor localization context, MDS can be applied to find a map of sensor positions (in 2-D or 3-D) when dissimilarities are measurements of range obtained, for example, via RSS or Time-of-arrival (TOA).

For the last 70 years many approaches to solving the MDS problem have been formulated (see Benzécri [1973]; Davidson [1983]; Greenacre [1984]; Cox and Cox [1994] and references therein). On the one hand, when the measured dissimilarities are equal to the true distances between sensors, classical MDS provides a closed formed solution by singular value decomposition of the centered squared dissimilarity matrix (see Section 3). On the other hand, when dissimilarities are measured in noise, other techniques should be used, usually based on iteratively minimizing a loss function between dissimilarities and distances. This framework encompasses techniques such as alternating nonlinear least squares (ALSCAL) [Takane et al. 1977], nonlinear least squares via majorizing functions (SMACOF) [Groenen 1993], nonmetric scaling [Kruskal 1964a, 1964b] or maximum likelihood formulations [Ramsay 1982; Zinnes and MacKay 1983]. Common to all these methods is the need for a central processing unit to gather all the available dissimilarities and perform the function minimization.

In contrast, we present a distributed MDS algorithm, which operates by minimizing multiple local loss functions. The local nonlinear least squares problem is solved using quadratic majorizing functions as in SMACOF. Since each local cost distributes additively over the network, each sensor contributes to the 
minimization of the global MDS loss function. In this way, our algorithm produces a sequence of position estimates with corresponding nonincreasing global cost and limited communication between sensors.

\subsection{Related Work}

Many aspects of the sensor localization problem have been addressed in recent literature. Notably, bounds on estimation performance have been derived for the cases when pair-wise measurements are RSS, TOA, Angle Of Arrival (AOA), or a combination [Moses et al. 2002, 2003; Patwari et al. 2003; Catovic and Sahinoglu 2004; Niculescu and Nath 2004]. Furthermore, centralized algorithms based on multidimensional scaling [Shang et al. 2003], convex optimization [Doherty et al. 2001], and maximum-likelihood [Moses et al. 2002] have demonstrated good estimation performance.

Research has also demonstrated the feasibility of distributed localization algorithms, which are required for scalability and balancing computational costs across large sensor networks. Distributed localization algorithms presented in the literature can be grouped into two types: adapted trilateration algorithms, and successive refinement algorithms. In the first type, devices estimate the distance to multiple known-location devices, using either a direct measurement, or if none exists, an estimate based on the shortest path to the known-location devices [Niculescu and Nath 2001; Savvides et al. 2002; Nagpal et al. 2003]. Then, using these range estimates to the anchors, the device uses trilateration to estimate its location. In the successive refinement approaches to localization, each device locally estimates its location from measured ranges to its neighbors. Each device begins with its own local coordinate system, and later merges it with neighboring coordinate systems [Čapkun et al. 2001]. The devices successively refine their location estimates [Albowicz et al. 2001; Savarese et al. 2001], effectively finding a solution to a global optimization problem that uses all ranges measured between neighbors.

The distributed algorithm presented here falls in the successive refinement category, which finds a minimum of a global cost function. In the dwMDS approach, however, the special cost function structure avoids the complicated step of merging local maps and a majorization algorithm ensures that each iteration decreases the global cost function. This global improvement is guaranteed even though sensors operate individual updates based only on information received from their few closest neighbors.

Although using a different formulation than the one proposed here, the following papers also apply MDS-type techniques to sensor localization:

-Plain MDS: In Shang et al. [2003], devices have connectivity information (whether or not two devices are in range). The distance between two connected nodes is defined to be 1 , while the distance between two nodes not in range is set to the number of hops in the shortest path between them (similar to Isomap [Tenenbaum et al. 2000]). The matrix of distances between each pair of devices is used by an MDS algorithm to estimate the coordinates of the devices. Compared to the present article, this centralized MDS method weights each distance equally. Unlike Shang et al. [2003], the method 
proposed here avoids the (usually inaccurate) estimation of distances between out-of-range sensors.

- Local MDS: In Ji and Zha [2004], a local version of MDS is used to compute maps of many local arrangements of nodes. These local maps are pieced together to obtain global maps. This method tends to perform better than the global MDS method when node density is nonuniform, or "holes" in coverage exist. The local calculations allow a distributed implementation, but weights are restricted to be either 0 or 1 . The formulation introduced in the present article removes that restriction, by allowing arbitrary non-negative weights, and naturally bypasses the complex step of fusing the local maps into a global map.

-Manifold Learning: Centralized manifold learning techniques are used in Patwari and Hero III [2004] to estimate sensor locations, without explicit range estimation, in cases where sensor data has correlation structure that is monotone in intersensor distance. Classical MDS is used to estimate physical location coordinates from the high-dimensional sensor data. The present article uses direct measurements of range between pairs of neighboring devices to estimate locations.

Common to most sensor localization methods, is the process of selecting sensor neighborhoods for range measurements. Most methods propose using only ranges measured between nearby neighbors, in order to limit communication costs and computational complexity. However, when ranges are measured with noise, the act of choosing neighbors based on these measurements will tend to select devices whose measured distances are shorter than the true distances. This article addresses this biasing effect and proposes a two-stage neighbor selection process that can be used to unbias location estimates even in high-noise environments. We remark that, to our knowledge, this problem has not been previously considered in the sensor localization literature.

\subsection{Outline}

The remainder of this article is organized as follows. Section 2 provides a formal statement of the sensor localization problem considered here. In Section 3 we describe the solution to the classical MDS formulation and discuss its shortcomings in a distributed and sensor network environment. The proposed algorithm is introduced in Section 4. In Section 5 we discuss statistical models for TOA and RSS measurements to show why a weighted MDS solution is important. Section 6 discusses the bias effect associated with using these noisy range measurements to select neighboring devices and proposes a solution. In Section 7 we show results on both simulated measurement data and on measured range data recorded for a 44-node sensor network in an indoor office environment. Finally, Section 8 concludes the article with a discussion about the proposed method, improvements, and future work.

\section{PROBLEM STATEMENT}

To be specific about sensor localization, we now formally state the estimation problem addressed in this article. 
Table I. Symbols Used in Text and Derivations

\begin{tabular}{|l|l|}
\hline Notation & \multicolumn{1}{c}{ Description } \\
\hline$D$ & Dimensions of location estimates $(D=2$ unless noted) \\
$N=n+m$ & Total number of sensors \\
$n$ & Sensors with imperfect or no a priori coordinate information \\
$m$ & Sensors with perfect $a$ priori coordinate knowledge ('anchor' nodes) \\
\hline$P_{i j}$ & Power received (dB) at sensor $i$ transmitted by sensor $j$ \\
$P_{t h r}$ & Minimum received power for successful reception \\
$d_{t h r}$ & Distance at which mean received power $=P_{t h r}$ \\
$d_{R}$ & Threshold distance for neighborhood selection \\
\hline $\boldsymbol{x}_{i}$ & Actual coordinate vector of sensor $i, i=1 \cdots n+m$ \\
$X$ & Actual coordinate matrix, $\left[\boldsymbol{x}_{1}, \ldots, \boldsymbol{x}_{n+m}\right]$ \\
$d_{i j}, d_{i j}(X)$ & Actual distance between sensors $i$ and $j$ in matrix $X$ \\
$\delta_{i j}^{(t)}$ & Range measured at time $t$ between sensors $i$ and $j$ \\
$w_{i j}^{(t)}$ & Weight given to the range measured at time $t$ between sensors $i$ and $j$ \\
$\bar{\delta}_{i j}$ & Weighted average measured range between sensors $i$ and $j$ \\
$\bar{w}_{i j}$ & Weight given to the average measured range between sensors $i$ and $j$ \\
\hline$S$ & Global objective function to be minimized \\
$S_{i}$ & Local objective function to be minimized at sensor $i=1 \cdots n$ \\
$\boldsymbol{x}_{i}^{(k)}$ & Estimated coordinates of sensor $i$ at iteration $k$ \\
$X^{(k)}$ & Estimated coordinate matrix at iteration $k$ \\
\hline
\end{tabular}

Consider a network of $N=n+m$ devices, living in a $D$-dimensional space ( $D=2$ or 3 , although the proposed formulation can handle arbitrary $D$ dimensional localization, as long as $D<N)$. Let $\left\{\boldsymbol{x}_{i}\right\}_{i=1}^{N}, \boldsymbol{x}_{i} \in \mathbb{R}^{D}$, be the actual vector coordinates of sensors, or, equivalently, define the matrix of coordinates $X=\left[\boldsymbol{x}_{1}, \ldots, \boldsymbol{x}_{n}, \boldsymbol{x}_{n+1}, \ldots, \boldsymbol{x}_{N}\right]$. The last $m$ sensors $(i=n+1, \ldots, N)$ have perfect $a$ priori knowledge of their coordinates and are called anchor nodes. The first $n$ sensors $(i=1, \ldots, n)$ have either no knowledge or some imperfect $a$ priori coordinate knowledge and are called unknown-location nodes. Imperfect $a$ priori knowledge about sensor $i \leq n$ is encoded by parameters $r_{i}$ and $\overline{\boldsymbol{x}}_{i}$, where, with accuracy $r_{i}, \boldsymbol{x}_{i}$ is believed to lie around $\overline{\boldsymbol{x}}_{i}$ (see Section 4 for a precise definition of these parameters). If no such knowledge is available, $r_{i}=0$. Summarizing, three distinct sets of sensors can be considered in this formulation based on their a priori information: perfect $(i>n)$, imperfect $\left(i \leq n, r_{i}>0\right)$, or zero coordinate knowledge $\left(i \leq n, r_{i}=0\right)$. Note that one or two of these sets might be empty, for example, no anchor nodes available and/or no prior information on sensors locations. These and other notations used throughout this article are gathered in Table I.

The localization problem we consider is the estimation of the coordinates $\left\{\boldsymbol{x}_{i}\right\}_{i=1}^{n}$ given the coordinates of the anchor nodes, $\left\{\boldsymbol{x}_{i}\right\}_{i=n+1}^{N}$, imperfect a priori knowledge, $\left\{\left(r_{i}, \overline{\boldsymbol{x}}_{i}\right)\right\}_{i=1}^{n}$ and many pairwise range measurements, $\left\{\delta_{i j}^{(t)}\right\}$, taken over time $t=1 \cdots K$. We use the terms 'dissimilarity' and 'range measurement' interchangeably, in order to seamlessly merge terms common to MDS and localization literature. The available range measurements $(i, j)$ are some subset of $\{1 \cdots N\}^{2}$. We assume that this subset of range measurements results in a connected network; otherwise, each connected subset should be considered individually. 
The method developed is general enough to adapt to any range measurement method, such as TOA, RSS, or proximity. We focus in particular on RSS-based range measurement, due to its desirability as a low-device cost method, but we also test the method using TOA range measurement in Section 7.

\section{CLASSICAL METRIC SCALING}

If we assume that we measure all the pairwise dissimilarities $\left\{\delta_{i j}\right\}_{i, j=1}^{N}$ between points, and that these correspond to the true Euclidean distances, then

$$
\delta_{i j}=d_{i j}=d\left(\boldsymbol{x}_{i}, \boldsymbol{x}_{j}\right)=\left\|\boldsymbol{x}_{i}-\boldsymbol{x}_{j}\right\|=\sqrt{\left(\boldsymbol{x}_{i}-\boldsymbol{x}_{j}\right)^{T}\left(\boldsymbol{x}_{i}-\boldsymbol{x}_{j}\right)} .
$$

By writing the squared distances as $d_{i j}^{2}=\boldsymbol{x}_{i}^{T} \boldsymbol{x}_{i}-2 \boldsymbol{x}_{i}^{T} \boldsymbol{x}_{j}+\boldsymbol{x}_{j}^{T} \boldsymbol{x}_{j}$, one can recover the matrix of inner products between points in the following way. Defining $\psi=\left[\boldsymbol{x}_{1}^{T} \boldsymbol{x}_{1}, \ldots, \boldsymbol{x}_{N}^{T} \boldsymbol{x}_{N}\right]^{T}$, the squared distance matrix, $D=\left[d_{i j}^{2}\right]_{i, j=1}^{N}$, can now be written as

$$
D=\boldsymbol{\psi} \boldsymbol{e}^{T}-2 X^{T} X+\boldsymbol{e} \psi^{T},
$$

where $\boldsymbol{e}$ is the $N$-dimensional vector of all ones. Defining $H$ to be the centering operator, $I-\boldsymbol{e} \boldsymbol{e}^{T} / N$, it follows that

$$
B=-H D H=H X^{T} X H .
$$

After multiplication with $H$, the columns of $X^{T} X$ have zero mean. Now, given $B$, one can recover matrix $X$, up to a translation and orthogonal transformation, as the solution to the following variational problem:

$$
\min _{Y}\left\|B-Y^{T} Y\right\|_{F}^{2}
$$

where $\|\cdot\|_{F}$ is the Frobenius norm and the minimum is taken over all $D \times N$ rank- $D$ matrices. The solution of (2) is given by

$$
X=\operatorname{diag}\left(\lambda_{1}^{1 / 2}, \ldots, \lambda_{D}^{1 / 2}\right) U^{T}
$$

where

$$
B=U \operatorname{diag}\left(\lambda_{1}, \ldots, \lambda_{D}\right) U^{T}
$$

is the (rank- $D$ ) singular vale decomposition (SVD) of matrix $B$.

The above derivation exposes the shortcomings of classical MDS. First, obtaining matrix $B$ requires the knowledge of all the pairwise dissimilarities, a scenario highly unlikely in a dense sensor network due to power and/or bandwidth constraints. Second, due to a lack of any special sparse structure, computing matrix $B$ and its SVD requires that all the dissimilarities be communicated and processed by a central processing unit, a communication-intensive operation in most sensor networks. Finally, (3) assumes that the true distances between points are available. For the realistic case in which range measurements are corrupted by multiplicative type noise (see Section 5), classical metric scaling minimizes the squared error between the squared distances $d_{i j}^{2}$ and $\delta_{i j}^{2}$ (rather than the distances themselves), which tends to amplify the measurement errors, resulting in poor noise performance. 


\section{DISTRIBUTED WEIGHTED MULTIDIMENSIONAL SCALING}

We propose a distributed weighted MDS algorithm (dwMDS) that fits the sensor networks framework of distributed computations and restricted communications and also accounts for measurement errors.

\subsection{The dwMDS Cost Function}

Motivated by the variational formulation of classical metric scaling (cf. (2)), we seek to estimate sensor positions by minimizing the following global cost function (a.k.a. STRESS function [Cox and Cox 1994]):

$$
S=2 \sum_{1 \leq i \leq n} \sum_{i<j \leq n+m} \sum_{1 \leq t \leq K} w_{i j}^{(t)}\left(\delta_{i j}^{(t)}-d_{i j}(X)\right)^{2}+\sum_{1 \leq i \leq n} r_{i}\left\|\boldsymbol{x}_{i}-\overline{\boldsymbol{x}}_{i}\right\|^{2} .
$$

where the actual Euclidean distance $d_{i j}(X)$ is given by (1), and we assume that for each pair $(i, j)$, up to $K$ dissimilarity measurements are available. The arbitrary weight $w_{i j}^{(t)}(t=1, \ldots, K)$ can be selected to quantify the predicted accuracy of measurement $\delta_{i j}^{(t)}$. If no such measurement is available between $i$ and $j$, or its accuracy is zero, then $w_{i j}^{(t)}=0$. We assume that $w_{i j}^{(t)} \geq 0, w_{i i}^{(t)}=0$ and $w_{i j}^{(t)}=w_{j i}^{(t)}$ : the weights are symmetric. Vector $\bar{x}_{i}$ contains prior information about the location of node $i(1 \leq i \leq n)$, while $r_{i}$ determines the influence of such information on the overall cost, by quantifying the accuracy of this prior location. If there is no prior information, then $r_{i}=0$. Note, function (4) differs from the standard MDS objective function in that we have added a penalty term to account for prior knowledge about node locations.

We stress that the variational formulation of (4) implies a nonparametric view of the location problem - the sensor positions are estimated by minimizing $S$ (w.r.t. $\left\{\boldsymbol{x}_{i}\right\}$ ), where no model assumptions are made about the statistical behavior of the observed dissimilarities. This permits the use of data-dependent weighting schemes (see Section 4.3), resulting in a cost function that can automatically adapt to different measurement models. Nevertheless, we remark that equation (4) can also be seen from a statistical viewpoint. Under a Bayesian perspective, (4) can be interpreted as the log posterior density of the node locations given the observed dissimilarities, $\log f\left(\left\{\boldsymbol{x}_{i}\right\} \mid\left\{\delta_{i j}\right\}\right)$, if we assume that the dissimilarities $\left\{\delta_{i j}^{(t)}\right\}$ are independently identically distributed Gaussian with mean $d_{i j}$ and variance $\left(2 w_{i j}^{(t)}\right)^{-1}$, and points $\left\{\boldsymbol{x}_{i}\right\}$ have a Gaussian prior with mean $\overline{\boldsymbol{x}}_{i}$, and variance $\left(2 r_{i}\right)^{-1}$.

After simple manipulations, $S$ can be rewritten as follows:

$$
S=\sum_{i=1}^{n} S_{i}+c
$$

where local cost functions $S_{i}$ are defined for each unknown-location node (ie. $1 \leq i \leq n)$,

$$
S_{i}=\sum_{\substack{j=1 \\ j \neq i}}^{n} \bar{w}_{i j}\left(\bar{\delta}_{i j}-d_{i j}(X)\right)^{2}+\sum_{j=n+1}^{n+m} 2 \bar{w}_{i j}\left(\bar{\delta}_{i j}-d_{i j}(X)\right)^{2}+r_{i}\left\|\boldsymbol{x}_{i}-\overline{\boldsymbol{x}}_{i}\right\|^{2},
$$


and $c$ is a constant independent of the nodes locations $X$. In (6), the $K$ weights and range measurements between $i$ and $j$ are summarized by a single weight $\bar{w}_{i j}=\sum_{t=1}^{K} w_{i j}^{(t)}$ and measurement $\bar{\delta}_{i j}=\sum_{t=1}^{K} w_{i j}^{(t)} \delta_{i j}^{(t)} / \bar{w}_{i j}$. As $S_{i}$ only depends on the measurements available at node $i$ and the positions of neighboring nodes: nodes for which $w_{i j}^{(t)}>0$ (for some $t$ ), it can be viewed as the local cost function at node $i$. We note that if $m=0$ (i.e., no anchor nodes are available) and $r_{i}=0$, for all $i$ (i.e., no prior information on the nodes locations), then $\partial S / \partial \boldsymbol{x}_{i}=2 \partial S_{i} / \partial \boldsymbol{x}_{i}$. This implies that the influence of $\boldsymbol{x}_{i}$ on the local cost $S_{i}$ determines its influence on the global cost $S$. Motivated by this cost structure, we propose an iterative scheme in which each sensor updates its position estimate by minimizing the corresponding local cost function $S_{i}$, after observing dissimilarities and receiving position estimates from its neighboring nodes.

\subsection{Minimizing the dwMDS Cost Function}

Unlike classical MDS, no closed form expression exists for the minimum of the cost function $S$ or $S_{i}$. By assuming that each node has received position estimates from neighboring nodes, we minimize $S_{i}=S_{i}\left(\boldsymbol{x}_{i}\right)$ iteratively using quadratic majorizing functions as in SMACOF (Scaling by MAjorizing a COmplicated Function [Groenen 1993]). This method has the attractive property of generating a sequence of nonincreasing STRESS values.

A majorizing function $T_{i}(\boldsymbol{x}, \boldsymbol{y})$ of $S_{i}(\boldsymbol{x})$ is a function $T_{i}: \mathbb{R}^{D} \times \mathbb{R}^{D} \rightarrow \mathbb{R}$ that satisfies: (i) $S_{i}(\boldsymbol{x}) \leq T_{i}(\boldsymbol{x}, \boldsymbol{y})$ for all $\boldsymbol{y}$, and (ii) $S_{i}(\boldsymbol{x})=T_{i}(\boldsymbol{x}, \boldsymbol{x})$. This function can then be used to implement an iterative minimization scheme. Starting at an initial condition $\boldsymbol{x}_{0}$, the function $T_{i}\left(\boldsymbol{x}, \boldsymbol{x}_{0}\right)$ is minimized as a function of $\boldsymbol{x}$. The newly found minimum, $\boldsymbol{x}_{1}$, can then be used to define a new majorizing function $T_{i}\left(\boldsymbol{x}, \boldsymbol{x}_{1}\right)$. This process is then repeated until convergence (see Groenen [1993] for details). The trick is to use a simple majorizing function that can be minimized analytically, for example, a quadratic function. Following Groenen [1993], we start by rewriting $S_{i}$ as:

$$
S_{i}\left(\boldsymbol{x}_{i}\right)=\eta_{\delta}^{2}+\eta^{2}(X)-2 \rho(X),
$$

where

$$
\begin{aligned}
\eta_{\delta}^{2} & =\sum_{\substack{j=1 \\
j \neq i}}^{n} \bar{w}_{i j} \bar{\delta}_{i j}^{2}+\sum_{j=n+1}^{n+m} 2 \bar{w}_{i j} \bar{\delta}_{i j}^{2}, \\
\eta^{2}(X) & =\sum_{\substack{j=1 \\
j \neq i}}^{n} \bar{w}_{i j} d_{i j}^{2}(X)+\sum_{j=n+1}^{n+m} 2 \bar{w}_{i j} d_{i j}^{2}(X)+r_{i}\left\|\boldsymbol{x}_{i}-\overline{\boldsymbol{x}}_{i}\right\|^{2}, \\
\rho(X) & =\sum_{\substack{j=1 \\
j \neq i}}^{n} \bar{w}_{i j} \bar{\delta}_{i j} d_{i j}(X)+\sum_{j=n+1}^{n+m} 2 \bar{w}_{i j} \bar{\delta}_{i j} d_{i j}(X) .
\end{aligned}
$$

Term (7) does not depend on $\boldsymbol{x}_{i}$ and term (8) is quadratic in $\boldsymbol{x}_{i}$. Only term (9) depends on $\boldsymbol{x}_{i}$ through a more complicated (sum of square roots) function. Define 
$T_{i}(\boldsymbol{x}, \boldsymbol{y})$ as:

$$
T_{i}\left(\boldsymbol{x}_{i}, \boldsymbol{y}_{i}\right)=\eta_{\delta}^{2}+\eta^{2}(X)-2 \rho(X, Y)
$$

where

$$
\rho(X, Y)=\sum_{\substack{j=1 \\ j \neq i}}^{n} \bar{w}_{i j} \frac{\bar{\delta}_{i j}}{d_{i j}(Y)}\left(\boldsymbol{x}_{i}-\boldsymbol{x}_{j}\right)^{T}\left(\boldsymbol{y}_{i}-\boldsymbol{y}_{j}\right)+\sum_{j=n+1}^{n+m} 2 \bar{w}_{i j} \frac{\bar{\delta}_{i j}}{d_{i j}(Y)}\left(\boldsymbol{x}_{i}-\boldsymbol{x}_{j}\right)^{T}\left(\boldsymbol{y}_{i}-\boldsymbol{y}_{j}\right) .
$$

Using the fact that, by the Cauchy-Schwarz inequality,

$$
d_{i j}(X)=\frac{d_{i j}(X) d_{i j}(Y)}{d_{i j}(Y)} \geq \frac{\left(\boldsymbol{x}_{i}-\boldsymbol{x}_{j}\right)^{T}\left(\boldsymbol{y}_{i}-\boldsymbol{y}_{j}\right)}{d_{i j}(Y)},
$$

it is easily seen that $T_{i}$ majorizes $S_{i}$. Minimizing $S_{i}$ through a majorizing algorithm is now a simple task of finding the minimum of $T_{i}$ :

$$
\frac{\partial T_{i}\left(\boldsymbol{x}_{i}, \boldsymbol{y}_{i}\right)}{\partial \boldsymbol{x}_{i}}=0
$$

An expression for this gradient is given in Appendix 8. If $X^{(k)}$ is the matrix whose columns contain the position estimates for all points at iteration $k$, one can derive an update for the position estimate of node $i$ using equation (12):

$$
\boldsymbol{x}_{i}^{(k+1)}=a_{i}\left(r_{i} \overline{\boldsymbol{x}}_{i}+X^{(k)} \boldsymbol{b}_{i}^{(k)}\right),
$$

where

$$
a_{i}^{-1}=\sum_{\substack{j=1 \\ j \neq i}}^{n} \bar{w}_{i j}+\sum_{j=n+1}^{n+m} 2 \bar{w}_{i j}+r_{i}
$$

and $\boldsymbol{b}_{i}^{(k)}=\left[b_{1}, \ldots, b_{n+m}\right]^{T}$ is a vector whose entries are given by:

$$
\begin{array}{ll}
b_{j}=\bar{w}_{i j}\left[1-\bar{\delta}_{i j} / d_{i j}\left(X^{(k)}\right)\right] & j \leq n, j \neq i \\
b_{i}=\sum_{\substack{j=1 \\
j=i}}^{n} \bar{w}_{i j} \bar{\delta}_{i j} / d_{i j}\left(X^{(k)}\right)+\sum_{j=n+1}^{n+m} 2 \bar{w}_{i j} \bar{\delta}_{i j} / d_{i j}\left(X^{(k)}\right) & \\
b_{j}=2 \bar{w}_{i j}\left[1-\bar{\delta}_{i j} / d_{i j}\left(X^{(k)}\right)\right] & j>n
\end{array}
$$

As the weights $w_{i j}^{(t)}$ are zero for nodes $j$ not in the relative neighborhood of node $i$, only the corresponding entries of vector $\boldsymbol{b}$ will be nonzero, and the update rule for $\boldsymbol{x}_{i}$ will depend only on this neighborhood (as opposed to the whole matrix $\left.X^{(k)}\right)$

We remark that, unlike the centralized SMACOF algorithm described in Groenen [1993], the computation of (13) does not require the evaluation of an $n \times n$ Moore-Penrose matrix inverse.

We also point out that the minimization algorithm proposed can be seen as a special case of optimization transfer methods through surrogate objective functions [Lange et al. 2000], which also include the popular EM algorithm. 


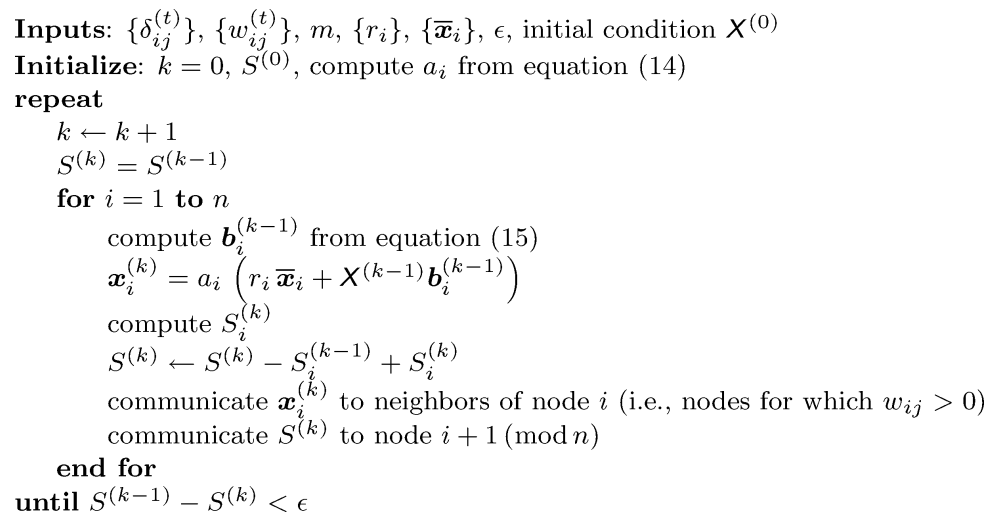

Fig. 1. Algorithm for decentralized weighted-multidimensional scaling.

\subsection{Algorithm}

The proposed algorithm is summarized in Figure 1. We make the following comments:

(1) The choice of weighting function $w_{i j}$ should reflect the accuracy of measured dissimilarities, such that less accurate measurements are down-weighted in the overall cost function. If a noise measurement model is available, $w_{i j}$ can be tailored to the variance predictions of the model. For example, one might select $w_{i j}=1 /\left(c_{1} \delta_{i j}+c_{2}\right)^{2}$ if the measurements are Gaussian distributed with standard deviation increasing linearly with the true distances: $\sigma=c_{1} d_{i j}+c_{2}$. When a reliable model is not available, one can adopt a model-independent adaptive weighting scheme. This is the approach adopted in this article. Inspired by the weighting frequently used in locally weighted regression methods (LOESS) [Cleveland 1979], we propose the following weight assignment:

$$
w_{i j}=\left\{\begin{array}{ll}
\exp \left\{-\delta_{i j}^{2} / h_{i j}^{2}\right\} & , \text { if } \delta_{i j} \text { is measured } \\
0 & , \text { otherwise }
\end{array},\right.
$$

where $h_{i j}=\max \left\{\max _{k} \delta_{i k}, \max _{k} \delta_{k j}\right\}$. This choice of $w_{i j}$, which equalizes the (nonzero) weight distribution in all sensors, has robust performance, as shown in the experiments reported in Section 7. Other weighting schemes are also possible, ranging from alternative monotone functions to a naive choice of unit weights for measured distances.

(2) The question of how to adaptively choose the neighbors of each node (which weights are made positive) in order to decrease communication costs or improve localization performance is addressed in Section 6.

(3) The values of $r_{i}$ should be chosen according to prior information. For example: if prior information about node $i$ was obtained using GPS, then $r_{i}$ should reflect the accuracy of the GPS sensor used; or, if anchor nodes are subject to small displacements, like vibrations, $r_{i}$ should quantify the mean squared error between the node's average position and the its expected movements. Under a Bayesian perspective, the choice of $r_{i}$ is analogous to the problem of subjective prior choice in a Bayesian model and thus can be guided by similar principles. Also, as in Bayesian problems, as more measurements per sensor 
are collected, the influence of the values of $r_{i}$ are discounted in the final solution. Section 7 includes a discussion on the influence of $r_{i}$ in experimental results.

(4) Regarding the initialization of the algorithm, every node requires an initial estimate of its position. This can be done using the algorithms proposed in Savarese et al. [2001] or Čapkun et al. [2001]: each node builds its local coordinate system, which is then passed along the network until a rough global map of the network is built. In the experiments reported in Section 7, we used a naive random initialization and found that the algorithm was robust with respect to these "rough" initial position estimates.

(5) In the description of the algorithm, it was assumed for notational convenience, that the algorithm cycles through the network in an ordered fashion (messages are passed between nodes in the order $1,2, \ldots, n$ ). However, many other noncyclic update rules are possible. In particular, one possibility is for (spatial) clusters of sensors to iterate among themselves until their position estimates stabilize. These estimates can then be transmitted to the neighboring clusters, before starting a new iteration step.

(6) Although the majorization approach used guarantees a nonincreasing sequence of STRESS vales, it may converge to a local minimum of this cost function, instead of the global one, like any gradient search method. This behavior can be alleviated to some extent by using some of the advanced search techniques proposed in Groenen [1993].

4.3.1 Computational Complexity and Energy Consumption. Regarding computational complexity, it is easily seen that the algorithm in Figure 1 scales as $O(n L)$, where $L$ is the total number of iterations required until the stopping rule is satisfied. This compares favorably to classical MDS, which requires $O\left(n^{2} T\right)$ operations, where $T$ is the number of steps required by the Lanczos method to compute the necessary SVD.

However, in sensor network applications, far more important than computational complexity, is the amount of communication required by the algorithm, as the energy consumed by a single wireless transmission can far outweigh the energy necessary for local computations. As we are interested in how communication complexity scales with the size of the network, we adopt the model proposed in Rabbat and Nowak [2004]. In this model, the average total energy used by a general data processing algorithm, as function of the number of nodes $n$, is given by

$$
\mathcal{E}(n)=b(n) \times h(n) \times e(n),
$$

where $b(n)$ is the average number of bits/packets transmitted, $h(n)$ is the average number of hops over which communication occurs, and $e(n)$ is the average amount of energy required to transmit one bit/packet over one hop.

For simplicity, we assume, in the following analysis, that the sensors are uniformly distributed over a square or cube of unit side length, for, respectively, a $D=2$ or $D=3$ dimensional network. The proposed algorithm requires that each node transmits its position estimate to other nodes from which it obtained range measurements. If we assume that a node is able to sense all other nodes within a threshold distance $d_{t h r}$, then the average number of neighbors a node 
can communicate with is upper bounded by $c_{1}(n-1) d_{t h r}^{D}$, where $c_{1}$ is the volume of the $D$-dimensional unit sphere (nodes close to the border of the unit square or cube actually have fewer expected neighbors). As this operation occurs for each iteration for every node, an upper bound on the average number of transmitted bits is $b_{\text {dwMDS }}(n) \leq O\left(n^{2} L d_{t h r}^{D}\right)$. Each communication to its neighbors can be made in one hop, so $h_{\mathrm{dwMDS}}(n)=1$. Thus, the average energy required for communication by the proposed algorithm is:

$$
\mathcal{E}_{\mathrm{dwMDS}}(n) \leq O\left(n^{2} L d_{t h r}^{D} e_{\mathrm{dwMDS}}(n)\right) .
$$

Notice that $e_{\mathrm{dwMSS}}(n)$ depends on $d_{t h r}$ (in a nonlinear way).

We remark that the same bound (17) on energy consumption is also valid for a sensor network with nodes distributed over a uniform grid of side length $O\left(n^{-1 / D}\right)$ (see Figure 3). This scenario makes it easier to compare the proposed method to a centralized algorithm, assuming a multi-hop communication protocol. To simplify the analysis, we consider the threshold distance $d_{t h r}=O\left(n^{-1 / D}\right)$. In this case, each node communicates only with its immediate neighbors in the uniform grid, making the average hop distance the same in the centralized and distributed cases. This implies that the same energy is required to transmit a bit/packet over one hop: $e_{\mathrm{dwMDS}}(n)=e_{\text {centr }}(n)$. For $d_{t h r}=O\left(n^{-1 / D}\right)$, each node will, on the average, receive range measurements from a fixed number of neighborhood nodes, no matter how big the network is. The centralized algorithm must transmit them to a fusion center. After a simple calculation, it can be shown that this results in $b_{\text {cebtr }}(n)=O(n)$ bits transmitted. For the uniform grid geometry, a simple calculation shows that the average number of hops from a node to the fusion center is $h_{\text {centr }}(n)=O\left(n^{1 / D}\right)$. Finally, we obtain the average energy required by a centralized algorithm,

$$
\mathcal{E}_{\text {centr }}(n)=O\left(n^{1+1 / D} e_{\text {centr }}(n)\right) .
$$

Substituting for the assumed $d_{t h r}$ in expression (17), we obtain the ratio between energies required by a centralized versus a distributed algorithm, in the uniform grid case:

$$
\frac{\mathcal{E}_{\text {centr }}(n)}{\mathcal{E}_{\text {dwMDS }}(n)}=O\left(\frac{n^{1 / D}}{L}\right)
$$

For dense networks of the same size $n$, and fixing a priori the maximum number of iterations allowed, a centralized algorithm will require an order of $n^{1 / D}$ more energy than the proposed distributed algorithm. Note that the costs of the centralized algorithm are not evenly distributed—nodes near the fusion center will disproportionately bear the forwarding costs.

To conclude this section, we remark that, for $D=2$ and $d_{t h r}=O\left(n^{-1 / D}\right)$, the proposed algorithm has a transport requirement of $O\left(n^{2} L d_{t h r}^{D}\right) \times d_{t h r}=$ $O(\sqrt{n})$ bit-meters/sec, which is the same as the transport capacity of a wireless network on a unit area region [Gupta and Kumar 2000]. This suggests that the implementation of the proposed algorithm is pratically feasible, even with more resource aggressive update rules (e.g., parallel updates of all nodes), for a large sensor network. 


\section{RANGE MEASUREMENT MODELS}

For concreteness, we assume throughout the rest of this article that range measurements between sensors are obtained either via RSS or TOA or a combination of the two. Both RSS and TOA can be measured via RF or by acoustic media; both media are subject to multipath and shadow fading phenomena, which impair range estimates.

\subsection{Time-of-Arrival}

For a TOA receiver, the objective is to identify the time-of-arrival (TOA) of the direct line-of-sight (DLOS) path. ${ }^{1}$ The power in the DLOS path is attenuated by any obstacles in between the transmitter and the receiver, and often, laterarriving non-line-of-sight (NLOS) multipath components arrive at the receiver with equal or greater power than the DLOS. As the distance between two devices increases, late-arriving paths contribute an increasing proportion of the overall received power. This increase has been observed in measured powerdelay profiles-for example, excess delay and RMS delay spread tend to increase with path length [Cox 1972; Hashemi 1993; Rappaport 1996]. Specifically motivated by radiolocation applications, researchers have used ns-synchronized measurement equipment to accurately identify the DLOS signal and show that NLOS signals' proportion of the total received power increases with path length [Pahlavan et al. 1998]. This NLOS signal power serves as self-interference, in combination with other noise and interference, which effectively decreases the SNR of the TOA measurements as the range increases. Previous research has suggested using weighted least-squares algorithms to improve localization performance [Chen 1999].

\subsection{Received Signal Strength}

Similarly, range measurements based on RSS degrade with distance. Objects in the environment between the transmitter and receiver have the effect of multiplying the signal energy by attenuation factors. The cumulative effect of many such multiplications, by a central limit argument, results in a log-normal distribution of RSS (or equivalently received power) at the receiver [Coulson et al. 1998]. If $P_{i j}(\mathrm{~mW})$, the received power in $\mathrm{mW}$ at sensor $i$ transmitted by sensor $j$, is log-normal, then received power in decibels, $P_{i j}=10 \log _{10} P_{i j}(\mathrm{~mW})$, is Gaussian. Furthermore, RF channel measurements have shown that the variance of $P_{i j}$ is largely constant over path length [Rappaport 1996; Patwari et al. 2003]. Thus $P_{i j}$ is typically modeled as

$$
\begin{aligned}
P_{i j} & \sim \mathcal{N}\left(\bar{P}_{i j}, \sigma_{d B}^{2}\right) \\
\bar{P}_{i j} & =P_{0}-10 n_{p} \log _{10}\left(d_{i j} / d_{0}\right)
\end{aligned}
$$

where $\bar{P}_{i j}$ is the mean power in decibel milliwatts at distance $d_{i j}, \sigma_{d B}^{2}$ is the variance of the shadowing, and $P_{0}(\mathrm{dBm})$ is the received power at a reference

\footnotetext{
${ }^{1}$ This is a different goal than for a communications receiver, which aims to synchronize to the time that maximizes the SNR, regardless of whether the signal power comes from the DLOS path or later arriving paths.
} 
distance $d_{0}$. Typically $d_{0}=1$ meter, and $P_{0}$ is calculated from the free space path loss formula [Rappaport 1996]. The path loss exponent $n_{p}$ is a parameter determined by the environment.

From this model for received power as a function of distance $d_{i j}$, the maximum likelihood estimator of distance is:

$$
\delta_{i j}=d_{0} 10^{\left(P_{0}-P_{i j}\right) /\left(10 n_{p}\right)} .
$$

If $P_{i j}=\bar{P}_{i j}$, then $\delta_{i j}=d_{i j}$. When $P_{i j} \neq \bar{P}_{i j}$, we can see why distance errors increase proportionally with distance. Consider a constant $\mathrm{dB}$ error in the received power measurement: $\Delta=\bar{P}_{i j}-P_{i j}$. For this error, $\delta_{i j}=d_{i j} 10^{\Delta /\left(10 n_{p}\right)}$, thus the actual distance is multiplied by a constant factor. In fact, the range estimation error, $\delta_{i j}-d_{i j}$, is directly proportional to $d_{i j}$ by the constant factor $10^{\Delta /\left(10 n_{p}\right)}-1$. Assuming constant standard deviation $\sigma_{d B}$ of received power with distance, the range estimation error standard deviation will also increase proportionally with distance.

This characteristic of RSS-based range estimation leads to a very high number of errors at large path lengths, which has limited its application in traditional location systems. However, in a dense sensor network, the distances between neighboring sensors is small, and a weighted least-squares estimator can be designed to fully utilize the accuracy of the range measurements made between the closest neighbors. A method for achieving this is proposed in the next section.

\section{ADAPTIVE NEIGHBORHOOD SELECTION}

Typically, neighbors are selected by choosing those devices that are closer than a threshold distance. But, since the exact distance is not known, we need to use noisy measurements to select neighbors. Range measurements, whether made via TOA, RSS, or proximity, are all subject to errors. In this section we discuss the biasing effects of selecting neighbors via noisy distance measurements, and how we can unbias the selection.

When distance is measured in noise, the act of thresholding neighbors based on the measured distance will tend to select the devices with smaller measured distances. For example, consider two devices separated by distance $R$, when $R$ is also the threshold distance. With some positive probability (due to noise), the measured distance, $\delta$, will be greater than $R$, and the two will not be considered neighbors. Alternatively, if $\delta \leq R$, the two will be considered neighbors, and $\delta$ will be used in the localization algorithm. The problem is that the expected value of $\delta$, for devices separated by $R$, which consider themselves neighbors, is less than $R$. Thus, the measured distance is negatively biased because of the effect of thresholding. Note that selecting the $K$-nearest-neighbors effectively has an adaptive threshold, and thus does not avoid this biasing effect.

This bias has not been specifically addressed in the sensor localization literature, because its effects are not severe in certain systems. Some proposed sensor localization systems measure very accurate distances, for example, using TOA in UWB or a combination of RF and ultrasound media-for these systems, the effect of selecting neighbors based on measured distances will be minimal. 
Alternatively, if neighbors are selected based on independent means (eg., based on RSS or connectivity when range estimates are based on TOA), then the biasing effect is avoided. ${ }^{2}$ Finally, when studies show results for the case in which all devices are connected to every other device, the thresholding step (and its biasing effect) is eliminated. In this article, we consider both noisy RSS measurements and small neighborhoods, so we cannot avoid the biasing effect. We limit our discussion to RSS measurements in this section, since low device costs and low energy consumption are very attractive device characteristics of RSS; but the discussion is also applicable to systems which use noisy TOA-based range measurements for neighbor selection.

\subsection{RSS-Based Biasing Effect}

When discussing thresholding based on RSS, we must make a distinction between the physical limits of the receiver and the threshold that we use to select neighbors, because generally, the two do not need to be the same. If a device has a large radio range in order to be robust to low device densities, it may want a stricter threshold when there are very many devices with which it can communicate. Denote $P_{t h r}$ to be the received power level below which a receiver cannot demodulate packets. (For most digital receivers with large frames and FEC, the frame error rate is very close to zero or very close to one for the vast majority of SNR, and the transition region is narrow. Thus, to a good approximation, for a constant noise level, we can state that packets above $P_{t h r}$ are received and demodulated correctly, while those below are not [Patwari and Hero III 2003].) Denote $P_{R}$ to be the received power level below which we do not include the transmitting device as a neighbor. Clearly, $P_{R} \geq P_{t h r}$. Equivalently, we can define distances $d_{t h r}$ and $d_{R}$ from (20) to be the range at which the mean received power is equal to $P_{t h r}$ and $P_{R}$, respectively.

Whether or not we select neighbors based on connectivity (measured power is greater than $P_{t h r}$ ) or select them based on a power threshold (measured power is greater than $P_{R}$ ), the biasing effect will be the same. In the following, we use $P_{R}$ and $d_{R}$ to indicate the thresholds (which may be set equal to $P_{t h r}$ and $d_{t h r}$ if desired).

Let $E\left[\delta_{i j} \mid P_{i j}>P_{R}\right]$ be the expected value of the range estimate between devices $i$ and $j$ given that the two are neighbors (i.e., the received power $P_{i j}$ is greater than $P_{R}$ ). Using the RSS measurement model (see Section 5.2), it can be shown that

$$
E\left[\delta_{i j} \mid P_{i j}>P_{R}\right]=\left\|x_{i}-x_{j}\right\| C \frac{1-\Phi\left(\sqrt{\beta} \log \frac{\left\|x_{i}-x_{j}\right\|}{d_{R}}+\frac{1}{\sqrt{\beta}}\right)}{1-\Phi\left(\sqrt{\beta} \log \frac{\left\|x_{i}-x_{j}\right\|}{d_{R}}\right)},
$$

where $\Phi($.$) is the cumulative distribution function of a standard Gaussian ran-$ dom variable, $\beta=\frac{10 n_{p}}{\sigma_{d B} \log 10}, C=\exp \left(\frac{1}{2 \beta^{2}}\right)$, and $\sigma_{d B}, n_{p}$ are channel parameters. Equation (22) is plotted in Figure 2 as a function of the ratio of the true

${ }^{2}$ Note, however, for the RSS/TOA example, that if both are available, we may wish to use a combination of both; and if not, RSS and TOA for a link, are correlated because objects in the environment tend to degrade both measurements simultaneously.

ACM Transactions on Sensor Networks, Vol. 2, No. 1, February 2006. 


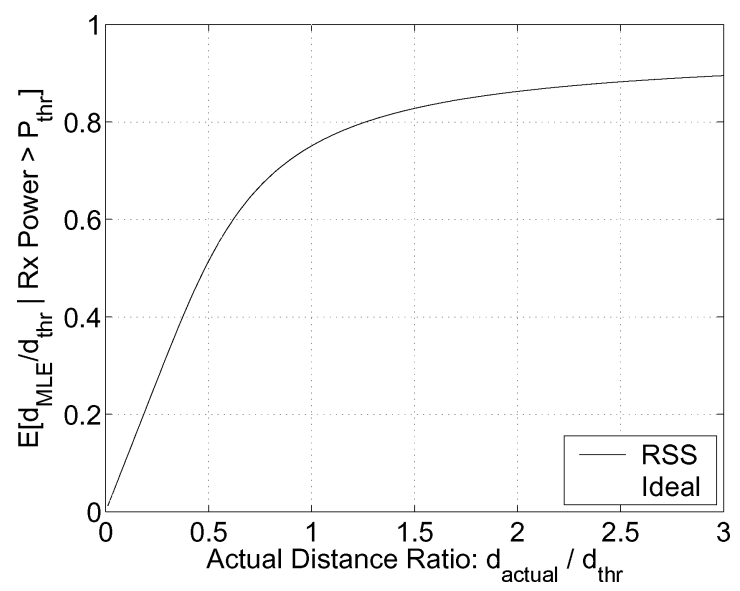

Fig. 2. The expected value of the RSS-based estimate of range given that the two devices are neighbors (- - ), and the ideal unbiased performance (-). The channel has $\sigma_{d B} / n=1.7$ and $d_{R}=1$ (or equivalently, distances are normalized by $d_{R}$ ).

distance to $d_{R}$. Ideally, the range estimator should have a mean value equal to the actual range. However, as the range increases, the expected value of $\delta_{i j}$ (given that $i$ and $j$ are neighbors) deviates from linear and asymptotically becomes constant. There is a strong negative bias for devices separated by $d_{R}$ or greater.

\subsection{Two-Stage Selection Algorithm}

Motivated by the negative bias phenomenon displayed in Figure 2, we propose a two stage neighborhood selection process, based on the predicted distances between sensors.

In the first step, the dwMDS algorithm from Figure 1 is run with a neighborhood structure based on the available range measurements: set $w_{i j}=0$ if $\delta_{i j}>d_{R}$. After convergence, this step provides an interim estimate $\left\{\hat{\boldsymbol{x}}_{i}\right\}$ of the sensors' locations. With high probability, the predicted distances between the estimated sensor locations will be negatively biased.

In the second step, these predicted distances from the estimated sensor locations are used to compute a new neighborhood structure, by assigning $w_{i j}=0$ if $\left\|\hat{\boldsymbol{x}}_{i}-\hat{\boldsymbol{x}}_{j}\right\|>d_{R}$. Some neighbors with low range measurements will be dropped, and some neighbors with possibly longer range measurements will be added. Then, using $\left\{\hat{\boldsymbol{x}}_{i}\right\}$ as an initial condition and the new neighborhood structure, the dwMDS algorithm is rerun, resulting in the final location estimates. Note that the predicted distances $\left\|\hat{\boldsymbol{x}}_{i}-\hat{\boldsymbol{x}}_{j}\right\|$ are used only to select neighbors (i.e., which weights are positive)— the measured ranges $\delta_{i j}$ are still used to determine the weight values.

We remark that this 2-step algorithm does not imply twice the computation. The dwMDS algorithm is based on majorization, and each iteration brings it closer to convergence. Since the first step only needs to provide coarse localization information, it does not need to be very accurate, and so the dwMDS 
algorithm can be stopped quickly with a large $\epsilon$. Next, the second step begins with very good (although biased) coordinate estimates, so the second run of the dwMDS algorithm will likely require fewer iterations to converge.

Note that for some of the devices that are considered neighbors in the 2nd run of the algorithm, the measured range $\delta_{i j}$ will actually be greater than $d_{R}$. Thus, to use this 2-step algorithm, $d_{R}$ must be sufficiently less than the physical communication limit of the devices, $d_{t h r}$, so that other range measurements can be considered. If we consider the noncircular (real-world) coverage area of a device, $d_{t h r}$ can be considered to be the mean radius of the coverage area, while $d_{R}$ should be set to the minimum radius of the coverage area.

\section{EXPERIMENTAL LOCALIZATION RESULTS}

We apply the proposed MDS algorithm to the location problem in a network, using both simulated data and real data collected on an experimental sensor network.

\subsection{Simulations}

In this section, all the simulated data were generated from the RSS measurement model presented in Section 5.2, with channel parameters $\sigma_{d B} / n_{p}=1.7$.

We first demonstrate the performance of the proposed algorithms on a network of $7 \times 7$ sensors arranged on a uniform grid of unit area, in which the four corner devices are anchor nodes and the remaining 45 are unknown location devices. For all experiments on this configuration, we use $d_{R}=0.4 \mathrm{~m}$ (yielding an average of 14 neighbors per device). We ran 200 Monte Carlo simulation trials to determine confidence ellipses, root-mean-square error (RMSE) and bias performance (per sensor) of the location estimates. The results are displayed in Figure 3, where we plot the mean and 1- $\sigma$ uncertainty ellipse of the estimator, and compare it to the actual device location and the Cramér-Rao lower bound (CRB) on the uncertainty ellipses, which was presented for the case of RSS measurements in Patwari et al. [2003]. We remark that the CRB shown is calculated assuming full connectivity (all devices measure range to all other devices), and as such provides only a loose lower bound on the best performance achievable by any unbiased estimator. In the first experiment, we provide a baseline best-case scenario by using perfect (noise-free) distance measurements to select neighborhoods. The baseline assumes that we have an oracle to tell us when the true distance between $i$ and $j$ is less than a threshold: $\left\|\boldsymbol{x}_{i}-\boldsymbol{x}_{j}\right\|<d_{R}$. This is shown in Figure 3(a), resulting in an RMSE of the location estimates of $0.090 \mathrm{~m}$ and an average bias of $0.019 \mathrm{~m}$.

For the second experiment, we remove the assumption of perfect connectivity knowledge. Instead, we use RSS measurements to select neighbors: devices $i$ and $j$ are neighbors if $P_{i j} \geq P_{R}$, or, equivalently, if $\delta_{i j} \leq d_{R}$. The results are shown in Figure 3(b). The estimates are strongly pulled towards the center of the square, due to the negative bias of the range estimates that are 'selected' by the connectivity condition. Now, the RMSE is $0.162 \mathrm{~m}$ and the bias is $0.130 \mathrm{~m}$.

A third experiment uses the adaptive neighborhood selection method proposed in Section 6.2. The results are displayed in Figure 3(c), where it can be 

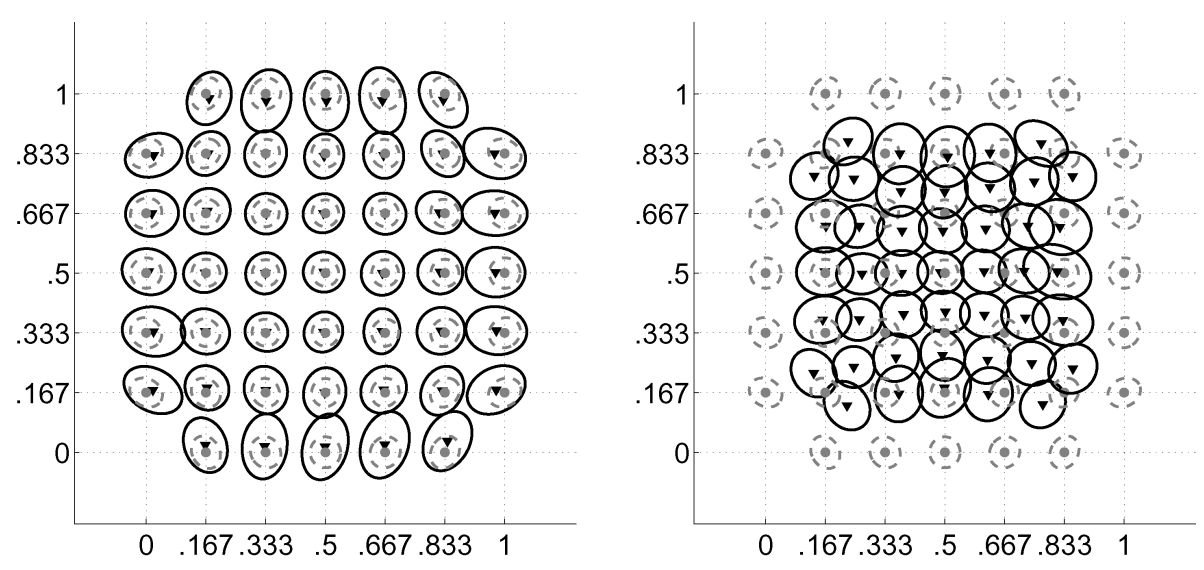

(a) Neighborhood selection using actual distances

(b) Neighborhood selection using measured ranges

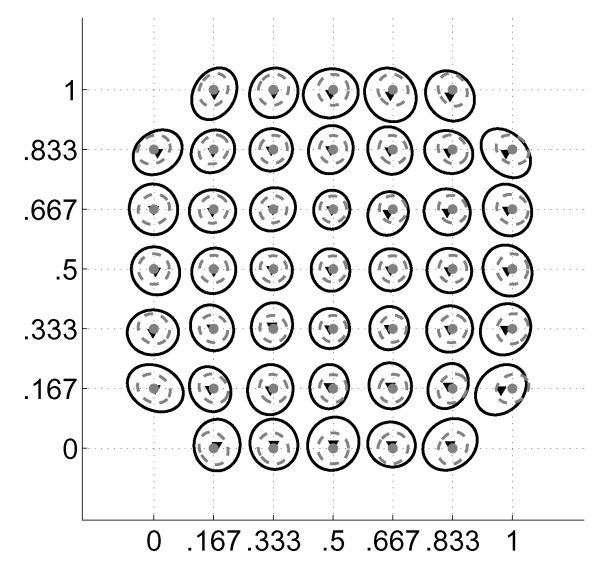

(c) Adaptive neighborhood selection

Fig. 3. Estimator mean ( $\mathbf{v})$ and 1- $\sigma$ uncertainty ellipse (-) for each blindfolded sensor compared to the true location $(\bullet)$ and CRB on the 1- $\sigma$ uncertainty ellipse (- - -).

seen that this method succeeds in removing the negative bias effect. The bias has gone back down to $0.012 \mathrm{~m}$, while the RMSE is $0.092 \mathrm{~m}$, just slightly higher than the baseline experiment using the oracle.

Comparing Figures 3(c) and 3(a), the localization errors of the two-step algorithm are spread more evenly throughout the network compared to the first experiment- the errors for edge devices are reduced, getting closer in magnitude to those in the center. Based on the similarity of the RMSE in both experiments, we believe that the 2-step process eliminates most of the neighbor selection bias. Additionally, by changing the neighbor lists (and therefore the weights) and rerunning the dwMDS algorithm, the 2 nd iteration also provides the opportunity to break out local maxima, which are more likely to affect edge devices. Finally, the low variance achieved by the 2 -stage algorithm is very close to the CRB, which no unbiased location estimator can outperform, despite the fact that the CRB is an optimistic bound for the scenario considered here. 


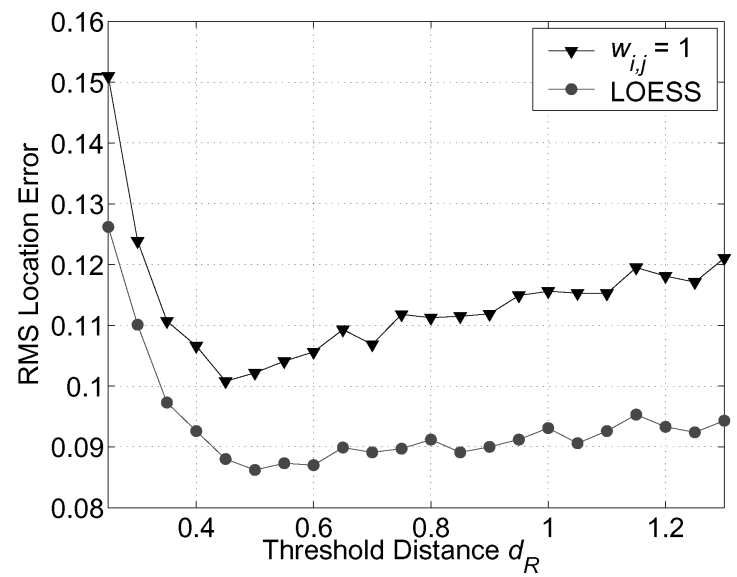

Fig. 4. RMSE versus threshold distance for the $7 \times 7$ uniform grid example using adaptive neighborhood selection, for different weighting schemes.

We also studied the influence of the threshold distance on the RMSE performance of the proposed algorithms. Figure 4 shows a plot of the RMSE vs. threshold distance (marked by ' $\bullet$ '), for the $7 \times 7$ uniform grid example using adaptive neighborhood selection. It can be seen that there is an optimal threshold distance, $d_{R}=0.5 \mathrm{~m}$, beyond which, no performance increase occurs. As $d_{R}$ is increased beyond this optimal value, more distant sensors are included in the cost function. By the RSS measurement model, the accuracy of range measurements degrades quickly with distance, thus adding these far a way sensors will not bring any gain to the estimation algorithm. Figure 4 also shows the behavior of the same quantities for the case of a naive weighting scheme: all measured distances have equal weights, while nonobserved distances have weights set to zero. The worse performance of this simple scheme shows further evidence to support the claim that weights should be chosen adaptively to reflect measurement accuracy, and further justifies the LOESS type weighting scheme (cf. (16)) adopted.

Finally, we show the influence of different choices of prior weighting $r_{i}$ in the quality of the localization solution. Under the same scenario, we now consider the four corner nodes to have imperfect information. In particular, the algorithm only has access to a noisy version of the actual coordinates of these nodes, perturbed by zero mean Gaussian noise with unknown variance $\sigma_{p}^{2}$. Figure 5 shows the resulting RMSE, obtained by running 5000 Monte-Carlo Simulation trials for $\sigma_{p}=0.025,0.050$, and 0.100, and setting $r_{i}=r$ for the corner nodes, where $r$ is made to vary between $10^{-2}$ and $10^{2}$.

From Figure 5, it can be observed that for small values of $r$, the RMSE levels off to a value that is constant across different values of $\sigma_{p}^{2}$. Essentially, the prior information is only being used to rotate and translate the final solution obtained by the MDS algorithm to best fit the estimated position of the anchor nodes. On the other hand, for high values of $r$, a similar phenomenon occurs, but this time due to the fact that the MDS algorithm is focusing on placing 


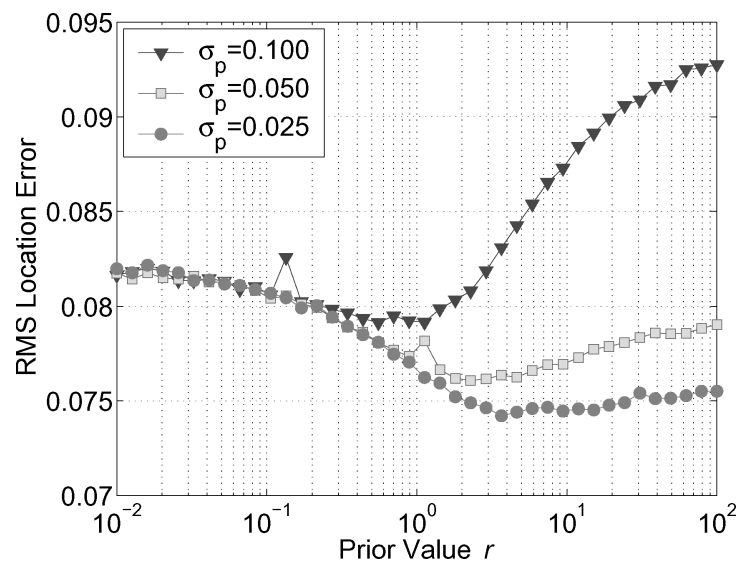

Fig. 5. RMSE versus prior weighting of the four corner nodes in the $7 \times 7$ uniform grid example using adaptive neighborhood selection.

sensors with prior information at their a priori coordinates, to the detriment of fitting range measurements.

For intermediate values of $r$, there is an optimal $r$ that best weights the relative information in the prior coordinates with respect to the weights chosen for the measured ranges. As we would expect, the optimal $r$ is inversely proportional to $\sigma_{p}^{2}$, although the exact dependency is influenced by factors such as the prior coordinates noise distribution, the weighting scheme chosen, and the number of neighbors of each node. Further research should investigate these dependencies. However, the RMSE near the optimal is a very shallow function of $r$-for all three curves, there is nearly an order of magnitude range within which the RMSE is within $1 \%$ of its minimum. So, although simulation might be necessary to find the optimal $r$, as long as $r$ is within the correct order of magnitude, the results will be nearly optimal. This suggest that, for little or no knowledge about the perturbations to prior coordinates used by the algorithm, choosing intermediate values of $r$ would be a good rule. In particular, this would result in a better RMSE than possibly using either:

- a method that uses prior coordinate information only to find the best rotation of a calculated relative map [Shang et al. 2003], which is analogous to low $r$ in the dwMDS method; or

-an MLE method which assumes that anchor coordinates are known perfectly

[Patwari et al. 2003], which is analogous to high $r$.

\subsection{Localization in a Measured Network}

To test the performance of the proposed algorithm on real-world channel measurements, we used the RSS and TOA measurements presented in Patwari et al. [2003]. This data set includes the RSS and TOA range measurements from a network of 44 devices (4 of which are anchor nodes) using a wideband direct-sequence spread-spectrum (DS-SS) transmitter and receiver pair operating at a center frequency of $2.4 \mathrm{GHz}$. The measurements were made in an open plan office building, within a $14 \times 14 \mathrm{~m}$ square area. The RSS between each 


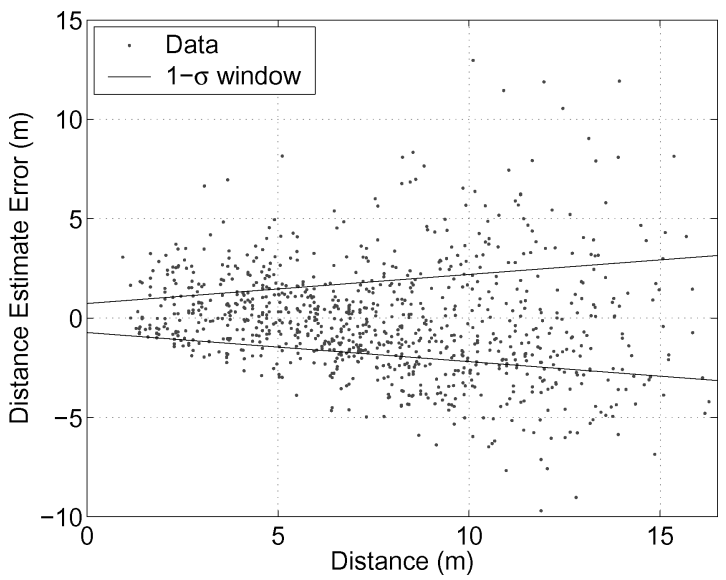

Fig. 6. Plot of distance measurement errors vs. distance. The $1-\sigma$ interval superimposed on the plot was obtained from the ML fit of the error measurement model $\mathcal{N}\left(d_{i j},\left(a d_{i j}+b\right)^{2}\right)$.

Table II. RMSE of Location Estimates in Experimental Network

\begin{tabular}{|c|c|c|c|}
\hline & Classical MDS & MLE [Patwari et al. 2003] & dwMDS \\
\hline RSS & $4.30 \mathrm{~m}$ & $\mathbf{2 . 1 8} \mathrm{m}$ & $2.48 \mathrm{~m}$ \\
\hline TOA & $1.96 \mathrm{~m}$ & $1.23 \mathrm{~m}$ & $\mathbf{1 . 1 2} \mathrm{m}$ \\
\hline
\end{tabular}

pair of devices was measured 10 times, from which the average was calculated and labeled as $P_{i j}$, for each pair $(i, j)$.

We use the bias-corrected MLE to estimate range from the RSS:

$$
\delta_{i j}=\frac{d_{0}}{C} 10^{\left(P_{0}-P_{i j}\right) /\left(10 n_{p}\right)} .
$$

We choose to divide by $C$ in (23) because this estimator, as opposed to the MLE in (21), is unbiased: $E\left[\delta_{i j}\right]=d_{i j}$. See Patwari et al. [2003] for details.

To give the reader a feeling of how challenging it is to do sensor localization using RSS range measurements in a real live scenario, we plot, in Figure 6, the error between range measurements and real distances: $\delta_{i j}-d_{i j}$. Note that the standard deviation of the RSS-based range estimator error increases steadily with distance. But, most importantly, the error as a percentage of actual range is often high: there are several range errors larger than $100 \%$ of the actual range.

We compare the performance of the dwMDS algorithm with adaptive neighborhood selection to classical MDS, and the MLE based solutions from Patwari et al. [2003]. Table II summarizes the RMSE of the location estimates. Figures 7(a) and 7(b) show the location estimates using classical MDS (which used all the pairwise range measurements between sensors) and the dwMDS algorithm, for the RSS measurement data set. The true and estimated sensor positions are marked by ' 0 ' and ' $\nabla$,' respectively, where the lines represent the estimation errors. The anchor nodes are marked with an 'x.' It can be observed that the dwMDS algorithm does much better than classical MDS. On the other hand, the RMSE of the dwMDS algorithm is slightly higher than the RMSE of the centralized MLE reported in Patwari et al. [2003]. However, that method 


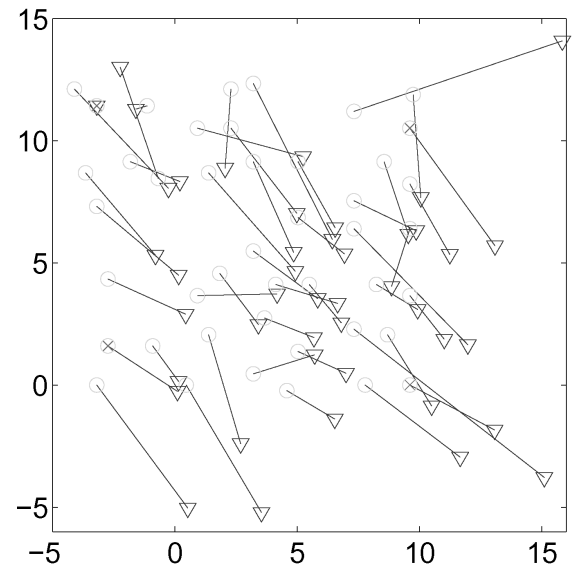

(a) Classical MDS (RSS)

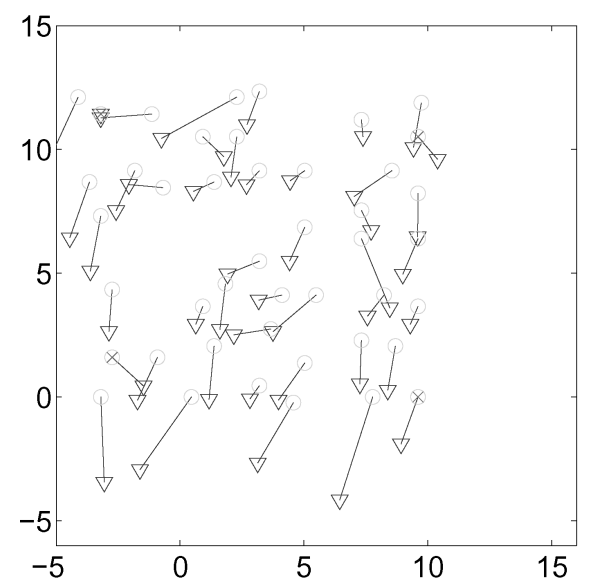

(c) Classical MDS (TOA)

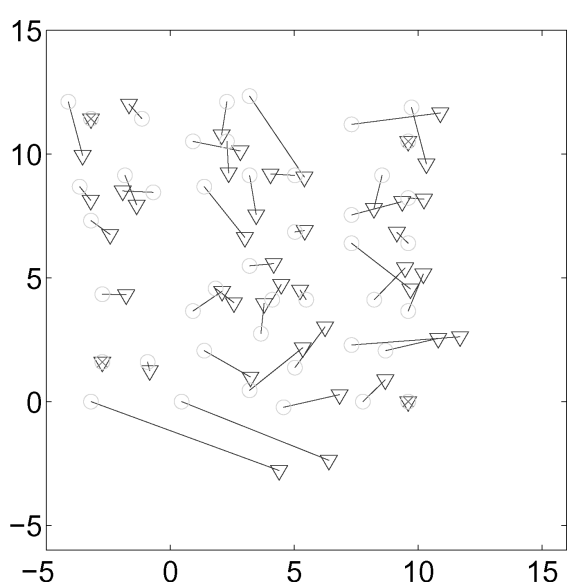

(b) dwMDS (RSS)

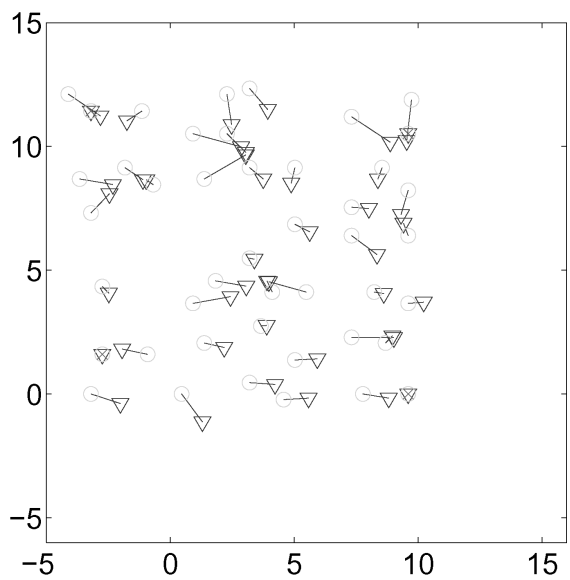

(d) dwMDS (TOA)

Fig. 7. Location estimates using RSS and TOA range measurements from experimental sensor network. True and estimated sensor locations are marked, respectively, by ' 0 ' and ' $\nabla$,' while anchor nodes are marked by 'x.' The dwMDS algorithm uses adaptive neighbor selection, with $d_{R}=6 \mathrm{~m}$.

not only uses all pairwise range measurements, but also relies on previously estimating the channel parameters. If we allow $d_{R}$ to increase at the expense of increasing communication costs, the dwMDS algorithm can reach an RMSE as low as $2.269 \mathrm{~m}$ for $d_{R}=8.5 \mathrm{~m}$.

Figures 7(c) and 7(d) show again the location estimates using classical MDS and the dwMDS algorithm, but this time for the TOA measurement data set. From Table II, it can be seen that the dwMDS algorithm outperforms all other location estimators. If we allow $d_{R}$ to increase at the expense of increasing communication costs, the dwMDS algorithm can reach an RMSE as low as $0.940 \mathrm{~m}$ for $d_{R}=7.5 \mathrm{~m}$. Once again, we stress that the dwMDS algorithm, unlike the MLE estimator from Patwari et al. [2003], does not use all the pairwise range measurements and does not assume knowledge of the distribution of the range measurements. 


\section{CONCLUSION}

This article proposes a distributed weighted-MDS method specially suited for node localization in a wireless sensor network. First, the method reflects the distributed nature of the problem, incorporating network communication constraints in its design. In this way, the need to transmit all range measurements to a central unit is eliminated, resulting in energy savings for a dense sensor network. Second, the inhomogeneous character of range measurements in a wireless network is accounted for by introducing weights that adaptively emphasize measurements believed to be more accurate. We stress that the dwMDS algorithm is nonparametric in its nature: it does not depend on any particular channel or range measurement models. This makes it applicable to a broad range of distance measurements, for example, RSS, TOA, proximity, without the need to tweak any parameters. We have shown via simulation that the algorithm has excellent bias and variance performance compared to the CRB, and that its performance in a real-world sensor network is similar to the centralized MLE algorithm.

We remark that the dwMDS algorithm can be applied more generally to dimensionality reduction problems across a network of processors, such as internet monitoring or distributed sensor data compression. To make it more general, other distance metrics can be used, such as $L_{p}(1 \leq p \leq 2)$ metrics. In this case, a majorization technique can still be used, which guarantees a nonincreasing cost function. For other general distances (without any convex structure), gradient descent techniques can be used. In particular, incremental gradient methods fit well the framework considered in this article, and might provide faster convergence rates at the cost of losing the monotonicity of the cost function. Other developments that can improve the algorithm's performance include extending the formulation to include nonrange measurements like AOA, or adding new terms to the cost function that model the correlations between range measurements.

\section{APPENDIX}

In this appendix, we give an expression for the gradient of the majorizing function $T_{i}$ defined by equation (10).

$$
\begin{aligned}
\frac{1}{2} \frac{\partial T_{i}\left(\boldsymbol{x}_{i}, \boldsymbol{y}_{i}\right)}{\partial \boldsymbol{x}_{i}} & =\left(\sum_{\substack{j=1 \\
j \neq i}}^{n} \bar{w}_{i j}+\sum_{j=n+1}^{n+m} 2 \bar{w}_{i j}+r_{i}\right) \boldsymbol{x}_{i}-\sum_{\substack{j=1 \\
j \neq i}}^{n} \bar{w}_{i j} \boldsymbol{x}_{j}-\sum_{j=n+1}^{n+m} 2 \bar{w}_{i j} \boldsymbol{x}_{j} \\
& -r_{i} \overline{\boldsymbol{x}}_{i}-\left[\sum_{\substack{j=1 \\
j \neq i}}^{n} \bar{w}_{i j} \frac{\bar{\delta}_{i j}}{d_{i j}(Y)}+\sum_{j=n+1}^{n+m} 2 \bar{w}_{i j} \frac{\bar{\delta}_{i j}}{d_{i j}(Y)}\right] \boldsymbol{y}_{i} \\
& +\sum_{\substack{j=1 \\
j \neq i}}^{n} \bar{w}_{i j} \frac{\bar{\delta}_{i j}}{d_{i j}(Y)} \boldsymbol{y}_{j}+\sum_{j=n+1}^{n+m} 2 \bar{w}_{i j} \frac{\bar{\delta}_{i j}}{d_{i j}(Y)} \boldsymbol{y}_{j} .
\end{aligned}
$$




\section{REFERENCES}

Albowicz, J., Chen, A., And Zhang, L. 2001. Recursive position estimation in sensor networks. In IEEE International Conference on Network Protocols. 35-41.

BenzÉCRI, J. 1973. L'Analyse des Données, Tome 2, L'Analyse des Correspondences. Dunod, Paris.

CAFFery JR., J. ANd Stuber, G. L. 1998. Subscriber location in cdma cellular networks. IEEE Trans. Veh. Tech. 47, 2 (May), 406-416.

ČAPKUn, S., HAMdi, M., AND HubaUx, J.-P. 2001. GPS-free positioning in mobile ad-hoc networks. In 34th IEEE Hawaii International Conference on System Sciences (HICSS-34).

CAtovic, A. AND SAhinoglu, Z. 2004. The Cramer-Rao bounds of hybrid TOA/RSS and TDOA/RSS location estimation schemes. Tech. Rep. TR2003-143, Mitsubishi Electric Research Laboratory. (Jan).

CHEN, P.-C. 1999. A non-line-of-sight error mitigation algorithm in location estimation. In IEEE Wireless Communication and Networking Conference. 316-320.

Cleveland, W. 1979. Robust locally weighted regression and smoothing scatterplots. J. Amer. Stat. Assoc. 74, 368, 829-836.

Correal, N. S., Kyperountas, S., Shi, Q., and Welborn, M. 2003. An ultra wideband relative location system. In IEEE Conference on Ultra Wideband Systems and Technologies.

Coulson, A. J., Williamson, A. G., and Vaughan, R. G. 1998. A statistical basis for lognormal shadowing effects in multipath fading channels. IEEE Trans. Veh. Tech. 46, 4 (April), 494502.

Cox, D. 1972. Delay doppler characteristics of multipath propagation at $910 \mathrm{MHz}$ in a suburban mobile radio environment. IEEE Trans. Antennas and Propagation AP-20, 5 (Sep), 625-635.

Cox, T. And Cox, M. 1994. Multidimensional Scaling. Chapman \& Hall, London.

Davidson, M. L. 1983. Multidimensional Scaling. Wiley, Ney York.

Doherty, L., Pister, K. S. J., And Ghaoui, L. E. 2001. Convex position estimation in wireless sensor networks. In IEEE INFOCOM. Vol. 3. 1655-1663.

Fleming, R. And Kushner, C. 1995. Low-power, miniature, distributed position location and communication devices using ultra-wideband, nonsinusoidal communication technology. Tech. rep., Aetherwire Inc., Semi-Annual Technical Report, ARPA Contract J-FBI-94-058. (July).

Girod, L., Bychkovskiy, V., Elson, J., AND Estrin, D. 2002. Locating tiny sensors in time and space: a case study. In IEEE International Conference on Computer Design. 214-219.

GreEnaCRE, M. J. 1984. Theory and Applications of Correspondence Analysis. Academic Press Inc., London.

GroenEn, P. 1993. The majorization approach to multidimensional scaling: Some problems and extensions. DSWO Press.

Gupta, P. And Kumar, P. R. 2000. The capacity of wireless networks. IEEE Trans. Inform. Theory $46,2,388-404$.

Hashemi, H. 1993. The Indoor Radio Propagation Channel. Proc. IEEE 81, 7 (July), 943-968.

JI, X. AND ZHA, H. 2004. Sensor positioning in wireless ad-hoc sensor networks with multidimensional scaling. In IEEE INFOCOM, 2652-2661.

KIM, S., PALS, T., Iltis, R., AND LEe, H. 2002. CDMA multipath channel estimation using generalized successive interference cancellation algorithm for radiolocation. In 37th Annual Conference on Information Sciences and Systems.

KRUSKAL, J. 1964a. Multidimensional scaling by optmizing goodness-of-fit to a nonmetric hypothesis. Psychometrika 29, 1-27.

KRUSKAL, J. 1964b. Nonmetric multidimensional scaling: a numerical method. Psychometrika 29, $115-129$.

Lange, K., Hunter, D. R., ANd Yang, I. 2000. Optimization transfer using surrogate objective functions. J. Computational Graph. Stat. 9, 1 (March), 1-20.

Moses, R. L., Krishnamurthy, D., and Patterson, R. 2002. An auto-calibration method for unattended ground sensors. In Proceedings of IEEE International Conference on Acoustic Speech and Signal Processing. Vol. 3. 2941-2944.

Moses, R. L., Krishnamurthy, D., and Patterson, R. 2003. A self-localization method for wireless sensor networks. EURASIP Journal on Applied Sig. Proc. 4 (Mar.), 348-358. 
Nagpal, R., Shrobe, H., And Bachrach, J. 2003. Organizing a global coordinate system from local information on an ad hoc sensor network. In 2nd International Workshop on Information Processing in Sensor Networks.

Niculescu, D. AND NATH, B. 2001. Ad hoc positioning system. In IEEE Globecom 2001. Vol. 5. $2926-2931$.

Niculescu, D. AND Nath, B. 2004. Error characteristics of ad hoc positioning systems. In ACM MOBIHOC.

Pahlavan, K., Krishnamurthy, P., and Beneat, J. 1998. Wideband radio propagation modeling for indoor geolocation applications. IEEE Comm. Magazine, 60-65.

Patwari, N. AND Hero III, A. O. 2003. Using proximity and quantized RSS for sensor localization in wireless networks. In IEEE / ACM 2nd Workshop on Wireless Sensor Networks \& Applications.

Patwari, N. and Hero III, A. O. 2004. Manifold learning algorithms for localization in wireless sensor networks. In Proceedings of IEEE International Conference on Acoustic Speech and Signal Processing.

Patwari, N., Hero III, A. O., Perkins, M., Correal, N., and O’Dea, R. J. 2003. Relative location estimation in wireless sensor networks. IEEE Trans. Sig. Proc. 51, 8 (Aug.), 2137-2148.

RABBAT, M. AND NowAK, R. 2004. Distributed optimization in sensor networks. In 3rd International Symposium on Information Processing in Sensor Networks (IPSN'04). Berkeley, CA.

RAMSAY, J. 1982. Some statiscal approaches to multidimensional scaling data. J. R. Statist. Soc. A 145, part 3, 285-312.

RAPPAPORT, T. S. 1996. Wireless Communications:Principles and Practice. Prentice-Hall Inc., New Jersey.

Savarese, C., Rabaey, J. M., ANd Beutel, J. 2001. Locationing in distributed ad-hoc wireless sensor networks. In Proceedings of IEEE International Conference on Acoustic Speech and Signal Processing. 2037-2040.

Savvides, A., Garber, W. L., Moses, R. L., and Srivastava, M. B. 2004. An analysis of error including parameters in multihop sensor node localization. IEEE Trans. Mobile. Comp. 4, 6, 567-577.

Savvides, A., Park, H., And Srivastava, M. B. 2002. The bits and flops of the n-hop multilateration primitive for node localization problems. In International Workshop on Sensor Networks \& Applications. 112-121.

Shang, Y., Ruml, W., Zhang, Y., And Fromherz, M. P. J. $2003 . \quad$ Localization from mere connectivity. In Proceedings of the 4th ACM International Symposium on Mobile Ad Hoc Networking \& Computing. 201-212.

TAKANE, Y., Young, F., AND DE LeEuw, J. 1977. Nonmetric individual differences multidimensional scaling: An alternating least squares method with optimal scaling features. Psychometrika 42, $7-67$.

Tenenbaum, J. B., DE Silva, V., And Langford, J. C. 2000. A global geometric framework for nonlinear dimensionality reduction. Science 290, 2319-2323.

ZINNES, J. AND MACKAY, D. 1983. Probabilistic multidimensional scaling: complete and incomplete data. Psychometrika 48, 27-48.

Received June 2004; revised January 2005 and June 2005; accepted July 2005 